\title{
On the Novelty of Nanotechnology: A Philosophical Essay
}

\author{
Joachim Schummer \\ Department of Philosophy, University of Darmstadt, Germany; js@hyle.org
}

\section{Introduction}

Nanotechnology has from its very beginning been surrounded with an aura of novelty. For instance, on the 28 introductory pages of the report that prepared the US National Nanotechnology Initiative (NNI), Nanotechnology Research Directions (NSTC/IWGN 1999), we read 73 times the term "new", 15 times "novel", 7 times "innovation", and 21 times "revolution". The authors concede that one should distinguish between different nanotechnologies, because "Many existing technologies do already depend on nanoscale processes. Photography and catalysis are two examples of 'old' nanotechnologies" (ibid, p. xxvi). One might conclude here that, if all the existing nanotechnologies are "old" nanotechnologies, "new" nanotechnologies do not yet exists but are only promises of the future. However, without further explanation and distinction between presence and future, they suggest that most nanotechnologies are or will be new. Furthermore, they claim that nanotechnology (in singular) is a generator of further new technologies, since "Nanotechnology will give birth to new fields that at present are only visions of leading researchers" (ibid., p. xviii).

Whenever science managers speak of nanotechnology (in singular), sophisticated distinctions seem to give way to plain claims about the present and future novelty of nanotechnology. As the NNI director Mihail Roco wrote in a 2001 report, "A revolution is occurring in science and technology [...] Nanotechnology will fundamentally transform science, technology, and society. In 10 to 20 years, a significant proportion of industrial production, healthcare practice, and environmental management will be changed by the new technology." (Roco \& Bainbridge, pp. 1, 19) When they put on their hats as science managers, scientists rarely reject but mostly support such novelty claim, as did for instance the chemists George Whitesides and Paul Alivisatos in the earlier report: "Nanostructures are the entry into a new realm in physical and biological science." (NSTC/IWGN 1999, p. 1) Backed by such support, scientific laypersons, from journalists to business consultants to ethicists, feel confident to deliver the novelty message to their respective audiences. For instance, up to the present day, news articles routinely speak of nanotechnology (singular) as a new technology, and ethicists usually introduce their papers on nanotechnology by emphasizing its novelty.

As the 1999 report illustrates, the issue of the novelty of nanotechnology has frequently come up, but dealt with only marginally in a way that is obscuring rather than clarifying. One reason, I assume, is that the general concept of novelty is, despite its frequent use, so complicated that its use typically invites misunderstandings. It seems appropriate therefore to keep the daily negotiations and rhetoric of novelty apart for a while, and ask what it actually means to say that something is novel. In this philosophical essay, I first try to clarify the different meanings of novelty and analyze the paradoxes and fallacies that dominate ordinary discourses on novelty. Equipped with these conceptual clarifications, I discuss novelty first in science and engineering in general and then in nanotechnology in particular by distinguishing between different levels and aspects of nanotechnology. The results allow reassessing public novelty claims about nanotechnology from an ethical and political perspective as well as some concluding remarks on the politics of controlling the production of novelty. 


\section{Kinds and Paradoxes of Novelty}

Compared to its frequent ordinary use, the concept of novelty is quite tricky - indeed full of paradoxes and fallacies lurking beneath the surface.

The first novelty paradox is that anything can be novel and non-novel at the same time. On the one hand, each and every event is novel because it has not yet happened before in exactly the same manner. Every moment differs from past and future moments, if we consider its location in time as unique. Since time flows irreversibly, as thermodynamics tells us, there are no two events that are identical. This is the trivial sense of novelty in which everything appears to be novel. On the other hand, there is "nothing new under the sun", to quote a book of the Bible written in the Greek tradition of skepticism (Ecclesiastes, 1:9-14). Everything happens within fixed constraints - laws of nature, if you want. Since these constraints define and circumscribe anything that is possible, nothing happens that really surprises those who know the constraints. This is the trivial sense of non-novelty in which nothing appears to be novel.

What then do we mean by singling out certain events as novel or new, while counting other events as old or recurring? Obviously we do not refer to the trivial meanings of novelty and non-novelty. While the trivial meaning of novelty highlights the uniqueness and individuality of every event, the trivial meaning of non-novelty focuses on its commonness and generality. Between both extreme views on the world, there is a broad range of considering events more or less individual or more or less general, because there is space to distinguish between uniqueness and commonness and thus between novelty and non-novelty. It all depends on our conceptual framework and the level of generality of our concepts. If we have different views, we may have an argument about the novelty of an event.

As with the novelty of events, the novelty of objects is very ambiguous and essentially depends on our conceptual framework. Assume you have discovered a flower and claim that this is a unique and novel one. Someone else may object that this flower is as any other flower so that there is nothing unique or novel about it (trivial non-novelty). So you refer to botanic taxonomy and explain that the flower is an exemplar of a new species that has never been described before. Your novelty claim thus turns out to be a claim of novelty of kind on a certain level of generalization, here the biological level of species. You take for granted that the flower is unique and novel as an individual entity (trivial novelty). At the same time you do not claim that your flower is an exemplar of a new genus, family, order, class, or division on the higher levels of the classificatory hierarchy of biology. You only claim that it is an exemplar of a new species. A more versed critic might object that your flower appears to be an exemplar of a new species only from a phenomenological point of view, whereas from a genetic standpoint, in terms of its DNA, it belongs to a well-known species. A skeptic might even maintain that the entire biological classification scheme, including the distinction between species, is an arbitrary way of imposing conceptual order on what in reality is a continuous variation in biological populations. In the end you have to defend a conceptual framework as naturally given in order to support your novelty claim.

Distinguishing something as novel thus depends on how you conceptualize it, which in turn depends on your conceptual framework. Strictly speaking you do not claim the novelty of an object, because the flower existed before. Instead, you claim that, within the existing conceptual framework of biology, you have made a novel conceptual discovery, which the flower only helped you to do. The alleged novelty of the object thus turns out to be the novelty of an event, of your own conceptual activity. Moreover, as the example of phenomenologically versus genetically founded classifications in biology illustrates, there are different ways of conceptualizing the world, depending on what one takes as more important. Any novelty claim is thus based on assumptions about what matters and what not. Novelty claims about objects in the world are thus hidden claims about the "truth" of conceptual frameworks and about the values that support the importance of that framework. 
Novelty claims that refer to an existing conceptual framework, such as the finding of a new species within the existing biological classification, are cases of normal novelty. Normal novelties simply fill a gap within an existing framework. Because the gap was pre-configured or even anticipated by the conceptual framework, normal novelties are not novel in a strict sense. Radical novelty, on the other hand, requires a different framework to be conceptualized and understood, what is sometimes called "thinking outside of the box". If you are unable to assume the different framework, because it is uncommon, unfamiliar, or foreign to you, you can neither comprehend the novelty nor appreciate it as novel. This poses a second novelty paradox: While normal novelty is not novel in a strict sense, because it was conceived before, radical novelty is incomprehensible.

Compared to the framework of biological taxonomy and other scientific classifications, which despite (or, historically speaking, because of) critics and skeptics are rather precise and robust, our ordinary concepts to describe the world are much more fuzzy, flexible, and guided by personal interest. In ordinary life disputes, claims about novel objects are frequently made in order to build a new, or considerably shift existing, conceptual frameworks in a way that suits best those who make these claims. To support such claims, a range of fallacies are available.

\section{Fallacies of Novelty}

Fallacies have an intellectual double-history. On the one hand, they are compiled in logic as typical forms of logical error. On the other, they are compiled in rhetoric as rhetorical means to convince people of something when sound arguments are missing. Understanding fallacies thus helps both avoid drawing wrong conclusions and protect against rhetorical seductions. Not surprisingly, several classical fallacies are developed around the concept of novelty.

The most famous one is the appeal to novelty (in Latin, argumentum ad novitatem). Something is considered good or important, or better and more important than something else, only by virtue of being novel. For instance, companies frequently advertise their products by emphasizing the newness, assuming that consumers have a strong preference for new products over old ones. If the appeal to novelty convinces many people such that they all want to have the new thing, the bandwagon fallacy further supports the appeal to novelty: according to that fallacy, the novelty is good only because everybody wants it. However, in general the appeal to novelty works only for certain audiences whose personal taste prefers modern things to traditional ones. Traditionalists, on the other hand, are often convinced by the counterfallacy, the appeal to tradition (argumentum ad antiquitatem). For instance, they consider something right just because it has ever been done so before and, accordingly, wrong if it is novel. In some specific areas, the appeal to novelty can be supported by arguments and thus is no fallacy proper. If a project has a clear definition of progress and works accordingly, the latest step is arguably better than the previous one. For instance, the latest computer anti-virus software that considers all available viruses is better than the previous version because it is more complete and thus safer. In general, however, the appeal to novelty relies on the idea of universal progress that every step in the course of time is an improvement, which in turn is frequently based on the related historiographical fallacy of novelty.

The historiographical fallacy of novelty relies on a naive way of writing history, which British call Whiggish history. In this view, the past is constructed so as to fulfill the goals of the present. Thus, everything of the past that does not suit the idea of a steady growth towards the current state, such as weird ideas, dead ends, and irresolvable conflicts, is simply ignored. In retrospect every novel step in history thus appears as improvement. From such a flawed history, people draw evidence for the claim that novelty is per se good which makes them subject to the appeal to novelty. However, one can similarly construct a history that focuses on weird ideas and dead ends and which would support the counter-claim according to which every novelty is a mistake. 
The appeals to novelty and tradition and the historiographical fallacy all draw unwarranted conclusion about the relevance of novelty. There are other fallacies that lead one to claim novelty where there is none. The two most frequent ones are the confusion of subjective and objective novelty and the confusion of term and object. Both fallacies are related to what Jean Piaget in his developmental psychology called egocentrism, i.e. that children are unable to distinguish between their perceptions and descriptions, on the one hand, and the world to be perceived and described, on the other. However, particularly if both fallacies come together, also many adults are prone to these fallacies which again can be fostered by the bandwagon fallacy.

Considering something novel only because one has never perceived or heard of it before, is committing the subjective/objective fallacy. Changing one's personal attention can thus generate novelties at will. However, the fallacy can easily be corrected by someone else arguing that the alleged novelty has been known by others before, which is how we usually learn to distinguish between subjective novelty and objective or intersubjective novelty. Things become more complicated if the alleged novelty comes with a new name by applying the term/object fallacy. Because language is a social institution that incorporates and conveys social knowledge, the new name suggests that the alleged novelty is also novel for anyone. If people are unable to distinguish clearly between terms and objects to be referred to by terms, the introduction of a new term is a powerful rhetorical tool to make novelty claims where there is none. And the more people adopt the new term, the more powerful becomes the rhetorical tool. Moreover, if the alleged novelty comes with a bunch of new terms that suggests a new conceptual framework, the term/object fallacy even allows one to introduce allegedly radical novelty.

The more diffuse the object is, the more difficult is it to identify the term/object fallacy. If the new term has an obscure meaning, such that it is hard to determine if it refers to a hitherto known or unknown object, the term/object fallacy is particularly powerful. On the other hand, introducing a new term for a diffuse range of known or unknown objects, in particular for abstract objects, can be semantically productive. As the Wittgensteinian theory of meaning suggests, the social establishment and continuous use of a new term may create and shape a new meaning. In that case, the use of the new term eventually creates a new object, such that the novelty claim works like a self-fulfilling prophecy.

We will see that most, if not all, novelty fallacies have played a role in the establishment of nanotechnology. Before discussing nanotechnology, however, it is useful to look at science and engineering in general, because these are unique fields of producing novelties.

\section{Novelty in Science and Engineering}

Despite the intricacy of the concept of novelty, there are only three cultural areas that seem to put strong emphasis on the production of novelty: news media, art, and science and engineering. ${ }^{1}$

The news media largely rely on the concept of trivial novelty, i.e. that any event is novel per se. However, an event qualifies to be reported as "news" only if it matters, if it meets the interests of the intended audience. This includes that the event happened only recently or was not known before, so that it is newly or freshly reported, because there is nothing worse for the media than old news. Therefore, the concept of newness in the news media refers to the report rather than to the event and to the subjective knowledge of the audience, such that the news media are prone to the subjective/objective fallacy. For instance, a wellestablished technology can be reported as news in the mass media if no other media has covered it before. Moreover, for the news media the antonym of new is old rather than common

1 One might be inclined to add fashion here. However, the recurrent stylistic repertoires and, indeed, the launches of "retro looks" clearly disqualify fashion from being a cultural area focused on novelty. Fashion works with relative novelty that depends on the time period after which consumers are willing to buy new products. 
or recurrent, which allows selling common or similarly recurrent stories as "news" so long as people are interested in. Hence, unlike what one might expect, novelty in the non-trivial sense does not play an important role in the news media.

That is entirely different in art and science, which are two cultural areas where the production of novelty is a necessary condition. Indeed, reproducing or imitating the works of others disqualifies people from being true artists or scientists - those who do so might even be accused of fraud. The concept of novelty in art is difficult, however, because artworks are, particularly in the formative arts and unlike the products of science, first of all unique objects that per se meet the criterion of trivial novelty. To which degree non-trivial novelty applies is a matter of theoretical interpretation and cannot be resolved here. It essentially depends on the conceptual level one is willing to consider in artworks.

In contrast, science and engineering are the only cultural areas in which novelty is produced and professionally managed on an international level, with strict measures to exclude the subjective/objective and term/object fallacies. This includes regular novelty checks of any research result as well as archives for storing previous results in a systematic manner to allow for quick novelty checks. In science, this is conducted by peers, particularly through the peer review procedure, who check research results for their epistemic reliability, disciplinary relevance, and novelty before their publication in professional journals. ${ }^{2}$ In engineering and applied science, where results are usually published as patents, national and transnational patent offices check patent filings for their non-obviousness or inventiveness, usefulness or industrial application potential, and novelty. In both cases the novelty is checked against previous publications, both articles and patents, which are indexed and archived in databases by professional information managers.

Both in science and engineering, trivial novelty does not matter because research results are not merely unique events but general results that must be reproducible by any other peer with the same research equipment. Such as an individual flower creates novelty only by virtue of being an exemplar of a hitherto unknown species, such creates an experimental research result non-trivial novelty only by being reproducible, by being an exemplar of all possible research results of the same kind. If, for instance, the research yields a piece of a hitherto unknown material, the novelty claim is about a new material species of which the piece is only a specimen. Similarly, if the research finds a causal relation between two parts of the individual experiment, the novelty claim is about the causal relation between the corresponding parts in any experiment of the same kind. In general, the novel finding must be communicable in professional language, i.e. by using general concepts that are known and well understood by peers and that precisely describe all the objects and operations of the experiments. That is to say that the novelty must be comprehensible within the established conceptual framework of the respective discipline. Such novelty thus meets the criterion of non-trivial normal novelty described above.

Following Thomas S. Kuhn's work on scientific revolutions and "paradigm changes" (Kuhn 1962), philosophers and historians of science have widely discussed radical novelty in science, when new results and their interpretation challenge the established conceptual framework to the point that they appear incomprehensible or even weird by peers. In standard evaluation procedures, such radical novelty can at first be hardly distinguished from so-called "pathological science" (Bauer 2002). If radical novelty in science comes with a fully developed alternative conceptual framework that is incompatible with the established one, the evaluation procedure is unable to make a rational, unbiased decision, because any evaluation would presuppose the preference of either conceptual framework. This means that by any rational standards, it is impossible to decide if radical novelty through scientific revolutions or

2 Interestingly, peer referees have a strong aversion to novelty rhetoric, i.e. they frequently criticize authors if they use terms such as "novel" or "new" in their manuscripts, instead of clarifying the novelty of their research by references to the pertinent literature (Daniel 1993). 
paradigm changes is an improvement over the established state of science. Historically, debates on radical novelties have been settled only by common social factors, i.e. by the distribution of power and rhetorical talent on either side. Rhetorical devices include various fallacies, such as the appeal to novelty and its counterpart, the appeal to tradition, discussed above. Yet, such arguments have no sound basis. Instead, and contrary to a widespread popular understanding, radical novelty in science through scientific revolutions or paradigm changes is neither good nor bad by rational standards.

Apart from research results, there are other aspects of science that can be novel in one sense or another. Most importantly, scientists frequently explore research areas that have been neglected before. Because every research explores something new, this means only that the research lies outside of the main focus of a discipline. However, disciplinary focuses can and do change in many regards. Because science has continuously grown over the past two centuries, with annual growth rates of about 5 per cent, disciplines are no static entities. They grow, split into subdisciplines, merge at their boundaries with other disciplines, give birth to new disciplines, and so on. Because the dynamics of disciplines does not essentially differ from the social dynamics of other populations, it is driven by common social factors rather than by the particularities of science. However, these social factors, which include internal and external forces, have an impact on the research areas in which scientific novelty is created.

Sometimes certain research results are considered to bear surplus novelty. In addition to being novel in the normal sense, they are said to induce further novelty in the future more than other research, as the quote on nanotechnology in the Introduction illustrates. Typical phrases that express this hope or promise include "key innovation", "groundbreaking", "land mark", "milestone", "revolutionary", and "cutting edge" research. One should be careful, however, with such phrases and claims if they are used to describe current research. Every research is meant to induce further research and thus further novelty, but the degree to which it will do so is largely unpredictable simply because science is unpredictable. Hence, those expressions are either used to make unwarranted predictions and promises or they function as rhetorical means to push the importance of certain research in the struggle for public attention and funding.

Now that we have clarified the different meanings and fallacies of novelty as well as the different levels and aspects of novelty in science and engineering, we are better prepared to discuss the novelty of nanotechnology.

\section{Scrutinizing the Novelty of Nanotechnology}

Because every published research result is supposed to meet the scientific requirements for normal novelty, we can assume that any published result in nanotechnology is novel in that regard. However, recalling the first novelty paradox, that does not mean that there is anything novel about nanotechnology, unless we find novelty on a more general level.

One of the most widely used definitions defines nanotechnology as the study of material structures in the scale of 1-100 nanometers in order to discover and exploit new properties of materials and devices that depend on the nanoscale structures for useful applications. ${ }^{3}$ However, almost any material happens to be structured at the nanometer scale in such a way that the structure essentially determines its properties. The definition perfectly describes the activity of most of chemistry since more than a century, as well as that of molecular biology,

\footnotetext{
3 For instance, the US committee on Nanoscale Science, Engineering and Technology (NSET) that launched the National Nanotechnology Initiative, defined nanotechnology as: "Research and technology development at the atomic, molecular or macromolecular levels, in the length scale of approximately 1-100 nanometer range, to provide a fundamental understanding of phenomena and materials at the nanoscale and to create and use structures, devices and systems that have novel properties and functions because of their small and/or intermediate size." (http://www.nsf.gov/home/crssprgm/nano/omb_nifty50.htm, retrieved in 2004).
} 
biochemistry, pharmacology, solid state physics, materials science and engineering, larger branches of electrical, chemical, mechanical engineering, and so on since several decades. Because the definition is much too broad, it makes nanotechnology a case of trivial nonnovelty.

The broadness of the definition suggests that "nanotechnology" is only an umbrella term that tries to encompass a multitude of distinct and long established science and engineering fields. Although the prefix "nano" is part of the old scientific standard nomenclature to describe a billionth of any measurement unit (such as in nanosecond, nanogram, or nanoOhm), scientists hardly used it before about 2000 to describe lengths other than wavelengths of light. Instead, because of some historical incidents, scientists used different units to describe the structure of materials and the size of particles, particularly Ångström ( 0.1 nanometer) and micron (1000 nanometer). Since huge national research budgets are available for nanotechnology, these conventions have suddenly changed such that the nanometer has largely replaced the other units. Because the units can simply be converted, that is only a change of terms without a change of meaning or object. Those who are unaware of the conversion, might fall victim to the term/object fallacy and assume new objects where only a new term was introduced. And because relabeling research nano has been a scientific mass movement, the bandwagon fallacy reinforces the term/object fallacy.

One might object that the broad definition of nanotechnology is only a conceptual clumsiness that should not be taken too seriously, because nanotechnology actually describes a range of very specific and novel research fields that cannot be put under a common definition by providing necessary and sufficient conditions. In this regard, nanotechnology as the total of these research fields is novel because each of the fields is novel. However, if there are no necessary and sufficient conditions to define nanotechnology, how are these research fields selected to belong to nanotechnology? The only way to select them by conceptual standards would be according to their novelty. That approach would seem to make nanotechnology novel by definition as the total of novel research fields at any time. Yet, because there has always been a set of novel research fields in the past, the set per se is not novel, even if we introduce a new name for it.

Moreover, the individual research fields that are nowadays called nanotechnology have a history that did not start in 2000 but goes back far into the 20th century if not earlier. The lack of history of science knowledge, and of scientific knowledge more general, makes people prone to the subjective/objective fallacy, such that they consider well-known things novel only because they have never heard of it before. A few examples of research fields that are all widely considered nanotechnology might illustrate that.

Chemical catalysis has been systematically studied and industrially exploited since the early 20th century, including for instance the use of size-tailored nanoscale pores in engineered zeolites since the 1950s (Sherman 1999). The history of molecular modeling started in the first half of the 20th century and then largely developed along with improvements of computer technology. Carbon nanotubes, which have become iconic of nanotechnology, were produced, studied, and characterized with transmission electron microscopy already in 1952 (Monthioux \& Kuznetsov 2006). Chemical vapor deposition, which was already known in the 19th century, has been used since the 1960s in the micro- and nanoscale production of films for ultra-thin coatings and semiconductor lithography, particles, and doted semiconductors (quantum dots) (Allendorf 1998). The production of molecular nano-devices, which until recently was called supramolecular chemistry, started in the 1970s (Lehn 1992, Balzani et al. 2003) as did genetic engineering, while atomic probe microscopy was developed in the early 1980s (Baird \& Shew 2004). A brief look into patent databases reveals that hydrogen storage systems, drug delivery systems (e.g., with liposomes, polymers, or cyclodextrins), and many other systems that are nowadays considered nanotechnology have been researched and heavily patented since the early 1970 s, although the drug delivery systems hardly ever came up to 
the expectations by passing clinical studies (e.g. Szejtli 1996). In addition, nanoparticles, nanocolloids, liquid crystals, polymers, nanocomposites, nanostructured materials, nanofilms, vesicles, and so on have been manufactured and studied for much of the 20th century, frequently following up 19th-century research and earlier artisan practices and products.

Of course in each of these fields, there is ongoing and important research that brings about new discoveries and inventions, as we should expect from any good research. However, that makes neither the individual research fields nor its total called nanotechnology new. It is true that some research fields have been neglected in the past, particularly the size and shape dependency of nanoparticle properties, and that more recently much stronger efforts have been made to exploit them for commercial purposes. But, again, that does not make the fields new. Instead, pouring more research money into these fields is meant to increase the rate of new discoveries and inventions, i.e. the rate of normal novelty within each of the fields, which brings them to public attention. One might object that there is a difference between research fields and technologies, arguing that although the research and development fields are old, they are meant to prepare new technologies in the sense of commercially available technological products and solutions. However, the argument hinges on the assumption that both can be clearly distinguished, whereas the current use of the term "nanotechnology" radically blurs the distinction. Even if we assume that there were a clear-cut distinction, it is not clear why the long-term research and patenting activities in these fields should now all of a sudden produce more of the long-wanted technological products, other than by suddenly increased research funding. In that case, the novelty claim would only be a promise or hope of future novelty in need of substantiation.

In sum, although each individual research result is novel in the normal sense, neither nanotechnology as a whole nor its individual research fields are new apart from the name. Since the individual research fields have developed continuously rather than revolutionary and changed only their names rather than their conceptual foundation, we can exclude both normal and radical novelty.

And yet, there is something new about nanotechnology that is not to be found on the research level but in the social organization of science. First and foremost, there is a new research budget in addition to, and mostly at the expense of, the established disciplinary budgets. Indeed, the national research funding agencies of industrialized countries, and increasingly of developing countries, have established a budget called "nanotechnology" - in Japan it was formerly called "atom technology". These budgets are largely earmarked for research across the disciplines with particular focus on bringing science and engineering closer together for the research and development of commercially useful products. Nanotechnology as a science policy idea stands for the goals of increased interdisciplinary research and for adjusting so-called fundamental research to the needs of applied science. More than just redistribution of money, the social reorganization of science requires scientists actively engaging in the movement for which they need to see new opportunities. To that end nanotechnology has from the very beginning been surrounded by, if not created from, visions, which were mostly derived from Eric Drexler's futuristic ideas of molecular scale robotics and other science fiction. For instance, the US national nanotechnology initiative has propagated that nanotechnology would bring about the next industrial revolution, unprecedented wealth, health, and security, that it will reshape the entire world atom-by-atom, and that it would allow enhancing human capacities beyond anything we have known before, even beyond our concept of what a human being is. Although visions have played an important role in the propagation of earlier science policy projects, from nuclear energy to genetic engineering, nano-visions have reached a new level regarding both their all-encompassing totality and futuristic dimensions.

Moreover, because science fiction writers had employed these visions under the name of "nanotechnology" long before scientists and science policy makers used the term, 
nanotechnology owns its origin from a new kind of interaction between science, science policy, literature, and the broader public. This includes also so-called investment consultants who have turned these visions into the next "big thing" for investors; journalists who have used the visions to write interesting stories about science; the techno-religious transhumanists who yearn for salvation by the help of molecular robotics; and ethicists who ponder on the ethical issues of futuristic, i.e. nonexistent, technology; and so on (Schummer 2004). The more the term "nanotechnology" has been used by a growing number of people, the more have the term/object fallacy and the bandwagon fallacy gained impact and the more has the conceptual distinction between visionary (but nonexistent) and new (and existent) been blurred.

We may conclude then that the novelty of nanotechnology lies in ideas about the future rather than in current technologies, beyond the usual production of novelty. On a social level, nanotechnology in this regard is a broad social movement that tries to direct and control current research towards these ideas. It is not about current novelty but about predictions and recommendations where the regular production of novelty by science and engineering will and should be focused in the future.

\section{The Ethics and Politics of Novelty}

Any new technology that provides solutions to hitherto unsolved societal problems has social and ethical dimensions, because the new opportunities, as well as the predictable and unpredictable risks and unwelcome consequences, need to be justly distributed. Therefore, the mere announcement, even the promise, of technological novelty, whether founded or not, induces both hopes and fears. Those who are considered experts and entitled to announce or promise novelty have political power with which they can deal responsibly or not. Three examples might illustrate the ethical and political dimensions of novelty: personal fears and hopes, regulatory issues, and international competition.

When nanotechnology is publicly propagated as a novel technology to solve whatever societal problem, that is frequently a misrepresentation that misleads the public, because the specific technology has usually been researched long before, and the novelty claim makes it neither more powerful nor novel other than by term/object and subjective/objective fallacies. Even if the novelty rhetoric is aimed at fostering public attention and research, such that it might become a self-fulfilling prophecy, that is a risky play on the people's hopes and fears. Imagine someone suffering from cancer or another serious disease in a state when existential decisions have to be made, for instance about a therapy with severe side effects. The promise of a forthcoming novel remedy by nanomedicine, as well as the omnipresent breakthrough rhetoric repeated by uncritical journalists, might have a crucial impact on such existential decisions. If the promise is unfounded propaganda, the person might be misled to make wrong existential decisions, for instance to postpone the therapy and wait for the new remedy and ultimately die because of the misinformation. Of course, novelty claims rarely affect life-anddeath decisions, but the case illustrates that they can do so and that playing on the hopes and fears of people is ethically relevant. On the other hand, suppressing novelty claims, for instance by referring to the trivial sense of non-novelty, can equally misinform and mislead decisions, such that it is ethically relevant too.

If a technology is said to provide revolutionarily new products and effects, only a fool would assume that these are all desirable and beneficial. As a rule, a new and unresearched, i.e. unknown, range of products and properties includes surprises, both welcome and unwelcome. If one announces the novelty of a range of products and properties, as has been done with nanoparticles, the novelty claim refers, strictly speaking, to both welcome and unwelcome effects. The logic of novelty thus requires pointing out both novel opportunities and novel risks of nanoparticles. And the more powerful the novel opportunities are praised, the more questionable are the received regimes of risk management. The novel opportunities of nanoparticles consist in the (hitherto neglected) exploration and exploitation of the range of 
properties that depend on the size and shape rather than on the chemical composition of the particles. While the phenomenon and its basic understanding is anything than new to scientists, the search for commercial applications of nanoparticles has drastically increased. However, all the regulatory frameworks for workplace safety and the production and marketing of chemicals just disregard the size- and shape-dependency of properties and identify the materials to be regulated only by chemical composition. Thus, a material that is well known and old in terms of its chemical composition but new in terms of its nanoparticle size and shape could be regarded safe in the legal framework even though it is toxic. Since nanoparticle products are already on the market, and many more are in the developmental state, our policy-makers follow a dangerous double strategy. They celebrate the novelty of nanotechnology in their funding and rhetoric, but irresponsibly stick to received and long outdated regulations.

We have seen that the science policy claims and promises of novelty should rather be understood as a means to direct and control the future production of novelties. On the international level, the promise of future novelty in a certain technological field might induce a competition among countries about who will be the first to make the breakthrough and harvest the economical potential. However, not every country has the international authority to make novelty promises and claims. Those who have the authority can always make sure they have a head start in the competition by selecting research fields for their novelty claims in which they are already particularly strong. Whereas those who lack the authority and are seduced by the promise to jump on the bandwagon will certainly lag behind. Thus, the authority to make novelty claims is a strong advantage in the international technological competition, because it allows determining international research agendas for one's own benefit.

As has been argued above, nanotechnology is neither in total nor in its individual fields novel, but it comes with the promise of future novelty that has even more aggressively been propagated as enabling the "next industrial revolution". Which economy would not want to be part of that? Following the US National Nanotechnology Initiative, numerous countries, both industrialized and developing, have launched similar nanotechnology initiatives and programs to engage in the competition. And most, if not all, have uncritically bought the novelty rhetoric and repeated it in their national agendas and pamphlets that are meant to inform the public. Although nanotechnology is particular because of its obscure definition, formerly hyped technologies, like information and biotechnologies, followed the same general pattern of international science policy leaders and epigones who are destined to lag behind. With each hype-cycle the hegemony of novelty claims thus helps reinforce the global technological and economic imbalance by producing winners and losers.

\section{Conclusion}

Science and engineering are unique in our society because they produce novelty on a regular basis in a highly professionalized manner that allows drawing clear distinctions between what is novel and what not. In contrast, ordinary language talk of novelty is prone to numerous confusions, paradoxes, and fallacies, which makes it an ideal rhetorical means in commercials. As a social movement, the propagation of nanotechnology has not only occurred at the interface between science and its publics, it has also merged the precise scientific meaning and the confused public meanings of novelty. In particular, science managers have spread the novelty claim about nanotechnology as a rhetorical means to direct future research, and thus to control future novelty production. Whether the political control of novelty production is possible at all and whether it leads to creative rather than monotonous research is still an open question. As a suddenly generated global mass movement it might favor opportunists rather than creative minds, both among scientists and science managers who prefer to follow the lead of others. By publicly spreading visions about possible future technological innovations in the disguise of novelty claims, science managers have gone beyond the usual financial incentives of science policy. While this might inspire some of those who actually produce nov- 
elty, it also affects ethical and political dimension of science and technology, because it stirs public hopes and fears, questions regulatory regimes, and induces international dynamics. The novelty talk thus reaches dimensions that science managers alone can hardly handle. Instead of making rhetorical novelty claims, they should better put the issue on the political agenda and ask in advance what kind of novelty people really want.

\section{References}

Allendorf, Mark: 1998, 'From Bunsen to VLSI: 150 Years of Growth in Chemical Vapor Deposition Technology', Interface (The Electrochemical Society), 7, no. 1, 36-39 [http://www.electrochem.org/dl/interface/spr/spr98/IF3-98-Pages36-39.pdf].

Baird, D. \& Shew, A.: 2004, 'Probing the History of Scanning Tunneling Microscopy', in: D. Baird, A. Nordmann \& J. Schummer. (eds.): Discovering the Nanoscale, IOS Press, Amsterdam, pp. 145-156.

Balzani, V.; A. Credi and M. Venturi: 2003, Molecular Devices and Machines: A Journey into the Nanoworld, Weinheim: Wiley-VCH.

Bauer, H.H.: 2002, 'Pathological Science' is not Scientific Misconduct (nor is it pathological)', Hyle: International Journal for Philosophy of Chemistry, 8, 5-20.

Daniel, H.-D.: 1993, Guardians of Science. Fairness and Reliability of Peer Review, Weinheim: VCH.

Kuhn, T.S.: 1962, The Structure of Scientific Revolutions, Chicago: University of Chicago Press.

Lehn, J.-M.: 1992, 'Supramolecular Chemistry - Scope and Perspectives: Molecules - Supramolecules - Molecular Devices' (Nobel Lecture 1987), in: T. Frängsmyr \& B.G. Malmström (eds.), Nobel Lectures, Chemistry 1981-1990, Singapore: World Scientific, pp. 444-491.

Monthioux, M. \& V.L. Kuznetsov: 2006, 'Who should be given the credit for the discovery of carbon nanotubes?', Carbon, 44, 1621-1623.

NSTC/IWGN. 1999. Nanotechnology Research Directions: Vision for Nanotechnology R\&D in the Next Decade, Washington DC.

Roco, M. C. \& W.S. Bainbridge (eds.): 2001. Societal implications of nanoscience and nanotechnology, Kluwer: Dordrecht.

Schummer, J.: 2004, "Societal and Ethical Implications of Nanotechnology': Meanings, Interest Groups, and Social Dynamics", Techne - Research in Philosophy and Technology, 8 (2), 56-87

Sherman, J.D: 1999, 'Synthetic zeolites and other microporous oxide molecular sieves', PNAS, 96 (7), 3471-3478 [http://www.pnas.org/cgi/content/full/96/7/3471]

Szejtli, J.: 1996, 'Historical Background', in: J.L. Atwood et al. (eds.), Comprehensive Supramolecular Chemistry, vol. 3 ('Cyclodextrins'), Oxford: Pergamon Press, pp. 1-3. 